\title{
EL DESAFÍO DE LA INNOVACIÓN AL INTERIOR DE LAS PYMES EN COLOMBIA Y COMO SOLUCIONARLOS MEDIANTE HERRAMIENTAS PRÁCTICAS
}

\author{
The challenges of innovation through the core of smes \\ in Colombia and how to solve them with practical tools
}

RICARDO VILLALBA ${ }^{1}$, ELKIN DARÍO MORALES GIL ${ }^{2}$

Recibido:02 de noviembre de 2018. Aceptado:12 de diciembre de 2018

DOI: http://dx.doi.org/10.21017/rimci.2019.v6.n11.a60

\section{Resumen}

En el presente artículo se tratarán los principales retos que enfrentan las PYMES en Colombia, mediante una recopilación de información a través de encuestas y datos ya recolectados por entidades gubernamentales, además de, algunos periódicos que se dieron a la tarea de analizar la situación actual de las PYMES en nuestra nación. Luego de esto, presentaremos algunas soluciones que resulten aplicables y eficientes para las PYMES.

Palabras clave: PYMES, sistemas, información, retos, Colombia, factoring, MOOC, TIC.
\end{abstract}

\section{Авstract}

In the following article we will talk about the main PYMES's challenges in Colombia, by means of a harvest of information through surveys and data collected by government agencies, besides, some newspapers that investigate about the actual situation of PYMES in Colombia. After this, we will present some solutions applicable and efficient by PYMES.

KeywordsPYMES, Colombia, systems, information, challenges, Colombia, factoring, MOOC, TIC.

\section{INTRODUCCIÓN}

$\mathrm{E}_{\mathrm{N}}$ ben buscar su supervivencia en el mercado, por ello, enfrentan grandes retos no solo a nivel nacional, sino también internacional, la globalización de los mercados que se ha experimentado desde el crecimiento acelerado de Internet, ha generado un cambio tan rápido en la economía global que muchas de las PYMES aún no se han adaptado al nuevo escenario económico, o bien, no saben cómo adaptarse a estos cambios, con el propósito de ayudar al crecimiento de las PYMES se desarrollarán los principales retos que enfrentan y los métodos mediante los cuales pueden abordarlos.

1 Docente Investigador del Grupo de Investigación OCA Operaciones Calidad Administración - Línea de investigación Diseño y gestión de la cadena de suministro de la Corporación Universitaria Republicana. Ingeniero de Producción, Universidad EAN, Especialista en Gerencia de Proyectos, Universidad EAN, actualmente cursando Maestría en Administración de Empresas, Universidad EAN. Correo electrónico: ricardoavillalbar@gmail.com

2 Estudiante de Ingeniería Industrial Semillero de Investigación de la Corporación Universitaria Republicana. 


\section{¿EN QUÉ SITUACIÓN SE ENCUENTRAS LAS PYMES EN COLOMBIA?}

Según los resultados de la GEP (Gran Encuesta PYME) realizada por la ANIF en el segundo semestre del 2016, se refleja una recesión en la evolución de las PYMES, esto es percibido por los empresarios debido a la disminución en la demanda de sus productos.

La ANIF destacó dos desafíos fundamentales para las PYMES en Colombia, las cuales son:

- Necesidad de mayor uso de las herramientas financieras y de innovación.

- La falta de internacionalización reflejada en la falta de exportación por parte de las PYMES.

La herramienta sugerida por la ANIF luego de realizar la GEP (Gran Encuesta PYME) es el factoring, dicha herramientas es usada por menos del 8\% de las PYMES según la ANIF y la explicaremos más adelante.

La internacionalización es un medio de crecimiento para las PYMES, y es un factor que afecta la economía del país en general, sin embargo, la falta de internacionalización de las PYMES se debe a las siguientes causas[1]:

- Entre un 30\% y 40\% de las PYMES no están interesadas en exportar

- Largos trámites legales y exigencia del cumplimiento de normas internacionales

- Costos asociados al proceso de exportación

- Desconocimiento del proceso para exportar

Otro hecho importante de mencionar es que de acuerdo ACOPI (Asociación Colombiana de Pequeñas y Medianas Industrias), del total de las exportaciones registradas por sus bases de datos en 2016, menos del $10 \%$ de estas fueron realizadas por PYMES, comparado a Europa en donde del total de las exportaciones es de alrededor del $40 \%$ son realizadas por PYMES.

En la revista "Mi pyme + productiva" publicada en mayo de 2017 por ACOPI en su edición
No. 1, estudia que en Colombia uno de los mayores retos que enfrentan las PYMES es la excesiva producción de resoluciones, decretos y leyes que no son sustanciales para el desarrollo económico del país, a continuación, enunciaremos algunos de los datos obtenidos por ACOPI acerca de toda la legislación emitida por el gobierno colombiano que afecta directamente a las PYMES[1]:

- Entre el 2000 y el 2016 se han emitido 94.748 actos administrativos.

- $73 \%$ (68.698) han sido resoluciones.

- $19 \%$ (17.168) han sido decretos.

- En 2016 se formularon 1.064 decretos de los cuales el 57\% son considerados no sustanciales.

Debido a la extensa legislación generada, las PYMES se ven obligadas a contratar asesorías para evitar sanciones legales, lo cual hace incurrir a las PYMES en sobrecostos y disminuyendo su capacidad de crecimiento.

A parte de esto, ACOPI explica que algunas de las normas emitidas por el gobierno colombiano se expiden sin una adecuada comunicación entre los ministerios implicados en las normas, lo cual genera que las empresas deben invertir gran parte de sus recursos y tiempo en cumplir con los requisitos de la legislación, hasta el punto de perder tiempo que pudieron haber usado en el desarrollo de sus actividades productivas.

ACOPI menciona los costos que asume una empresa para exportar en Colombia en comparación al resto de América Latina y los países pertenecientes a la OCDE (Organización para la Cooperación y el Desarrollo Económico)[2]:

- En Colombia una empresa debe invertir en promedio 112 horas para el cumplimiento de inspecciones aduaneras e inspecciones obligatorias en los puertos, lo cual se traduce en un costo aproximado de 545 USD por cada exportación.

- Este proceso en el resto de América Latina cuesta 547 USD por cada exportación

Rev. Ingeniería, Matemáticas y Ciencias de la Información Vol. 6 / Núm. 11 / enero - junio de 2019; pág. 113-120 
- Y en los países pertenecientes a la OCDE lleva 12 horas y cuesta 150 USD por cada exportación

Posteriormente ACOPI nos brinda una gráfica donde se muestran las principales razones por las cuales, las PYMES no exportan en mayor cantidad[3]. Fig. 1.

Otro de los retos que enfrentan las PYMES según la revista semana, es que, desde hace 15 años aproximadamente, expertos fijaron su atención en los deficientes resultados de la parte administrativa de las empresas alrededor del mundo, llegando a la conclusión de la necesidad inmediata de fortalecer el llamado "Gobierno Corporativo" [4].

Sabiendo que la mayoría de las empresas en Colombia, son conformadas como sociedades, el gobierno colombiano a través de Confecámaras, la cámara de comercio de Bogotá y la superintendencia de sociedades desarrollaron los documentos llamados[5]:

- “Guía colombiana de gobierno corporativo para sociedades cerradas y de familia
- "Hacia la implementación de buenas prácticas de gobierno corporativo en sociedades cerradas"

Estos documentos son una herramienta de apoyo, pensada principalmente para los empresarios de las PYMES que buscan optimizar la capacidad de sus empresas mediante las buenas prácticas del gobierno corporativo como:

- Dirección

- Toma de decisiones

- Administración de riesgos

- Solución de conflictos entre socios

A parte de esto, el siguiente reto de las PYMES según ACOPI, es el uso de las TIC para ingresar al mercado electrónico y relacionarse con clientes internacionales, donde más del $30 \%$ siguen sin hacer uso de estas herramientas.

Para este reto ACOPI sugiere el uso de la primera red social de comercio exterior para PYMES

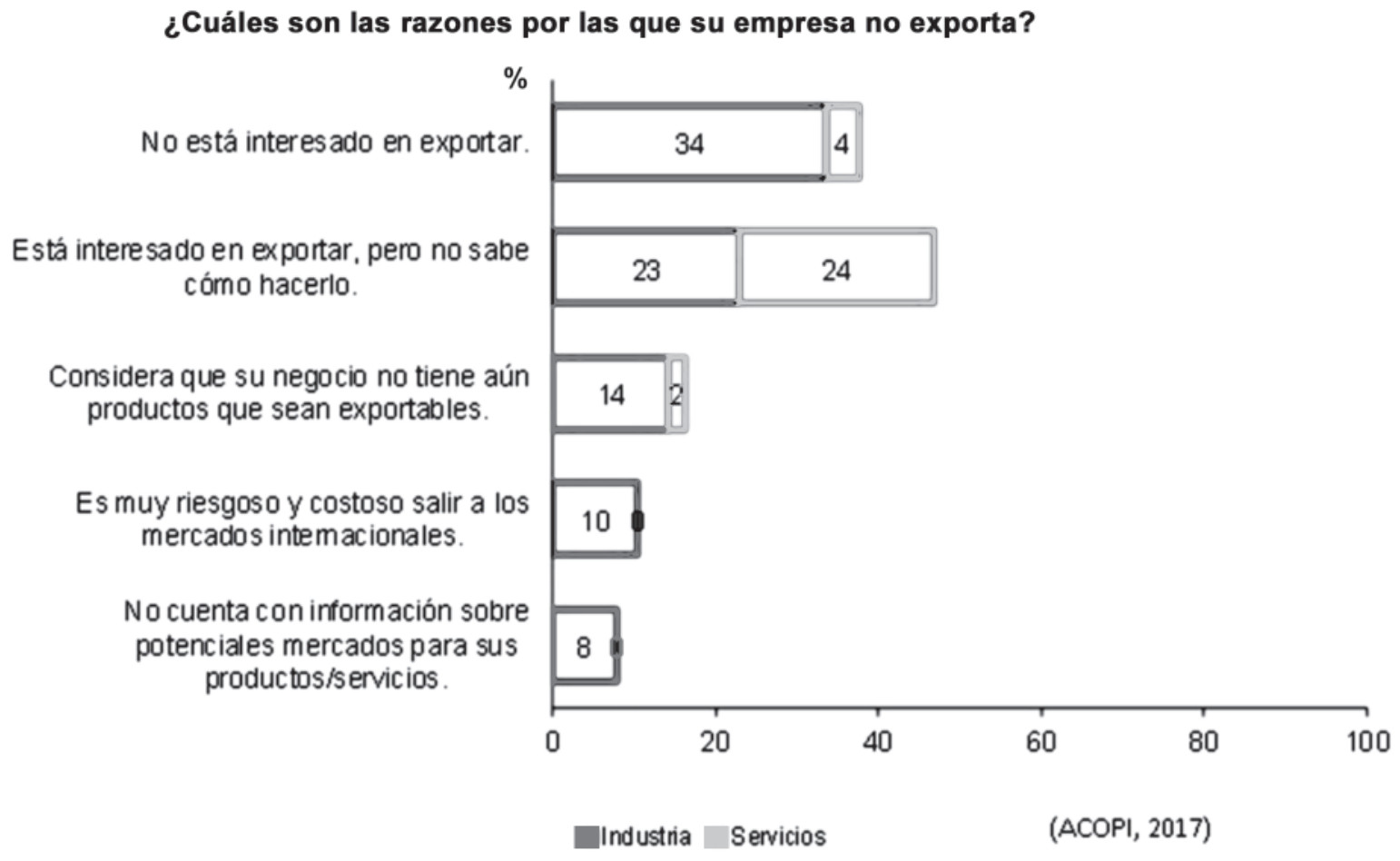

Fig. 1. Cuales son las razones por las cuales las empresas no exportan. 
llamada "Connect Americas", esta plataforma la explicaremos en detalle más adelante.

Sin embargo, la forma en que las PYMES enfrenten estos retos será la clave para su supervivencia, ahora listaremos los retos que hemos recopilado, los cuales son:

1. Uso de herramientas financieras

2. Liderazgo como pilar de la innovación

3. Internacionalización

4. Gobierno corporativo

5. Uso del Networking y cursos a través de internet

Ya conociendo los principales retos, se desarrollará cómo abordar cada uno de estos retos.

\section{1) Uso de herramientas financieras}

Las PYMES en Colombia que comercializan sus productos bajo la modalidad de crédito a 30, 60 o 90 días, suelen llegar a tener problemas de liquidez en algún momento.

No es posible que los clientes paguen esas facturas antes de los plazos establecidos, por lo cual, las PYMES pueden recurrir al factoring como medio para solucionar el problema de liquidez.

\section{1) ¿Qué es el factoring?}

Es una herramienta financiera altamente aprovechada por las grandes empresas, pero, no muy usada por las PYMES en Colombia[6].

Consiste en un crédito que realiza una entidad bancaria usando como soporte las cuentas por cobrar de la empresa en vez de sus estados financieros.

Es decir, en vez de que la entidad bancaria haga un préstamo haciendo un estudio de crédito tradicional, lo hace soportándose en las cuentas por cobrar de las empresas.

El factoring ofrece las siguientes ventajas:
- Más económico que un préstamo tradicional en comparación a las tasas de interés del crédito tradicional.

- Se obtiene el dinero de la cuenta por cobrar antes de la fecha acordada.

- La entidad financiera se encarga de hacer el cobro de la cuenta por cobrar que soporta la operación de factoring.

- La empresa no adquiere una deuda con la entidad bancaria.

- Se soporta en las facturas y no en los estados financieros.

- El monto del factoring es adicional a la capacidad de endeudamiento de la empresa Para ilustrar mejor la operación de factoring, expondremos un ejemplo de dicha operación:

Una empresa que se dedica a la fabricación de juguetes vende a un cliente un lote por valor de $\$$ 70.000.000 COP a 90 días, sin embargo, la empresa requiere el dinero lo más pronto posible para pagar los gastos fijos de la compañía.

Para ello se dirige a los 5 días de generar la factura a 90 días, a una entidad bancaria con el servicio de factoring y el resultado es el siguiente:

- El banco cobra una comisión del 1.5\% del valor de la cuenta por cobrar, es decir, le cobra a la empresa $\$ 1.050 .000$ COP por la operación.

- La empresa recibe $\$ 68.950 .000$ COP en el día 6, es decir, obtiene liquidez 84 días antes de lo previsto.

- La entidad bancaria cobra la factura al cliente en el día 90.

- La empresa no adquirió ninguna deuda por la operación.

Esta herramienta financiera, les brindaría a las PYMES obtener el dinero de las cuentas por cobrar antes de lo previsto, para hacer crecer sus empresas.

Rev. Ingeniería, Matemáticas y Ciencias de la Información Vol. 6 / Núm. 11 / enero - junio de 2019; pág. 113-120 


\section{2) Liderazgo como pilar de la innovación}

Otro reto para las PYMES es encontrar alguna herramienta de innovación, la que proponemos en este artículo es el "Brainstorming" también conocida como "Tormenta de ideas".

Las personas que trabajan diariamente en las PYMES colombianas conocen mejor que nadie sus propios trabajos, de esta manera se puede aprovechar las ideas que los empleados tengan para lograr una mayor eficiencia de las operaciones, mediante un "Brainstorming" el empresario deberá organizar reuniones con su equipo de trabajo y escuchar atentamente los problemas que tienen los empleados en sus lugares de trabajo, $y$, las oportunidades de crecimiento que tienen gracias a las ideas de su equipo.

Para lograr esto el empresario PYME, debe, adquirir la capacidad de liderazgo con el fin de aprovechar al máximo la creatividad de su equipo de trabajo, esto lo puede lograr al dominar los siguientes pilares del liderazgo, planteados en el libro "Piense y hágase rico" de Napoleón Hill[7], un autor que realizó una investigación de más de 20 años sobre los empresarios y líderes más exitosos de su época, llegando a 11 factores claves para un liderazgo efectivo:

1. Valor inquebrantable (confianza en sí mismo)

2. Autocontrol

3. Sentido de justicia

4. Precisión en las decisiones

5. Exactitud en los planes

6. Habito de hacer más de lo que le corresponde

7. Personalidad agradable

8. Simpatía y comprensión

9. Dominio del detalle

10.Dispuesto a asumir la responsabilidad total

11. Cooperación (trabajo en equipo)

\section{3) Internacionalización}

Para muchas PYMES colombianas, la idea de expandir sus nichos de mercado al exterior del país resulta una tarea aterradora, sin embargo, las estadísticas de Confecámaras muestran que alrededor del 70\% de las PYMES que deciden comercializar en el exterior sobreviven a sus primeros 10 años de operación, mientras que las PYMES que deciden satisfacer solo la demanda interna del país, sobreviven alrededor del $50 \%$.

Por ello ACOPI en la segunda edición de la revista "Mi Pyme + productiva" publicada en septiembre de 2017, nos comparte que ProColombia a través de la iniciativa Futuexpo en alianza con la DIAN, el programa de transformación productiva (PTP) y Bancóldex ponen a disposición de los empresarios de las PYMES los programas de formación para construir los pilares para la internacionalización, los cuales son[8]:

- Calidad (Certificaciones)

- Innovación

- Investigación y desarrollo

- Cumplimiento de tiempos de entrega

- Seguimiento de los envíos

Para usar la herramienta de diagnóstico los empresarios de las PYMES deben ingresar a la página de Procolombia y buscar la opción "Ruta exportadora" o pueden seguir el siguiente link[9]:

- http://www.procolombia.co/rutaexportadora/

Esta herramienta le ayudara a las PYMES en el diagnóstico del potencial exportador de sus empresas, o bien, a encontrar y explorar como lograr productos o servicios que sean exportables.

ProColombia pone a disposición de todos los interesados en exportar, las herramientas y asesoría que requieren para aumentar el número de PYMES exportadoras en Colombia. 


\section{4) Gobierno corporativo}

Para explicar la importancia de un gobierno corporativo, primero debemos definirlo, por lo tanto, usaremos la siguiente definición dada por Adrian Cadbury en el foro mundial sobre gobierno corporativo en 2003[10].

“En su sentido más amplio, el gobierno corporativo consiste en mantener el equilibrio entre los objetivos económicos y los sociales entre los objetivos individuales y los comunitarios. El marco de gobierno se establece con el fin de promover el uso eficiente de los recursos y, en igual medida, exigir que se rindan cuentas por la administración de esos recursos. Su propósito es lograr el mayor grado de coordinación posible entre los intereses de los individuos, las empresas y la sociedad. El incentivo que tienen las empresas y sus propietarios y administradores para adoptar las normas de gestión aceptadas a nivel internacional es que ellas los ayudarán a alcanzar sus metas y a atraer inversiones. En el caso de los Estados, el incentivo es que esas normas fortalecerán sus economías y fomentarán la probidad de las empresas"

Confecámaras a través de su página web[10], explica los beneficios que se obtienen de la implementación de un gobierno corporativo y son los siguientes:

\section{a) Mayor acceso al financiamiento}

Los inversionistas y entidades bancarias suelen invertir o prestar dinero, a las empresas que demuestran un sólido equipo de trabajo reflejado en un buen gobierno corporativo, ya que, se genera confianza a través un trabajo profesional

\section{b) Mayor valorización de las empresas}

Un adecuado gerenciamiento de una empresa lleva a un crecimiento económico, por lo cual, un gobierno corporativo es clave para dirigir adecuadamente los recursos disponibles.

\section{c) Mejor desempeño organizacional}

Se obtiene una mejora en las políticas laborales y en la administración de los recursos, lo cual se refleja en un mejor ambiente laboral para los integrantes de la empresa.

\section{d) Menor riesgo de crisis financieras}

Al tener un equipo analizando la situación de la empresa periódicamente, se logra detectar posibles crisis financieras en el futuro y tomar acciones que prevengan estos eventos.

\section{e) Mejores relaciones con las partes interesadas}

Para mejorar las relaciones de la empresa con los empleados, bancos, socios entre otras personas o entidades involucradas con la empresa, se vuelve clave el aprendizaje de un liderazgo efectivo, básicamente el gobierno corporativo debe estar compuesto por personas que tengan la capacidad de comunicarse efectivamente con todo tipo de personas o entidades.

Confecámaras sabe que muchos de los empresarios de las PYMES necesitan una herramienta que les ayude en el proceso de implementación del gobierno corporativo en sus organizaciones[10].

Para esto los empresarios pueden ingresar al portal de implementación de gobierno corporativo a través de[10][11][12]: http://gc.confecamaras. co/front/.

Lo que debe hacer es registrarse e iniciar sesión en el portal, luego de esto, la herramienta de implementación del gobierno corporativo lo ira guiando paso a paso, para que logre dicha implementación.

Pero también, Confecámaras ofrece un apoyo especializado para aquellos empresarios que necesitan el apoyo de un equipo de profesionales que los guíen en la implementación del gobierno corporativo, la cual pueden solicitar a través de la plataforma de Confecámaras.

\section{5) Uso del Networking y cursos a través de internet}

Para los empresarios que dirigen a diario las PYMES del país, la capacitación resulta algo vital si quieren hacer crecer sus negocios, por lo cual brindaremos herramientas digitales que pueden usar como apalancamiento para ellos y sus negocios[13].

La primera herramienta que brindaremos serán los MOOC (Masive Online Open Courses), bá- 
sicamente son cursos masivos dictados a través de aplicaciones como EDX y Coursera, o, a través de páginas web desde algún ordenador.

Gran parte de las universidades de todo el mundo ofrecen algunos de sus cursos de manera gratuita y otros de pago, a través de los MOOC, esto permite que cualquier persona que desee capacitarse tenga una herramienta que le guie en su proceso de aprendizaje.

La segunda herramienta que brindaremos es TED, una aplicación en la cual se recopilan conferencias de gente exitosa en su campo, las cuales comparten susexperiencias y conocimientos a través de esta plataforma digital.

Luego de esto debemos explicar que es el Networking y cómo usarlo como medio para ingresar al comercio electrónico o e-commerce.

El Networking es una red de contacto entre personas o empresas, que permiten compartir dar a conocer el talento de las personas o los productos o servicios que ofrece una empresa.

Esta herramienta posibilita el intercambio de información y contactos, así como el establecimiento de relaciones entre los usuarios en busca de un bien común.

La red social empresarial que recomendaremos para las PYMES en este artículo académico es "Connect Americas", que tiene el propósito de ayudar a las pequeñas y medianas empresas de Latinoamérica y el Caribe a realizar más transacciones de comercio exterior e insertarse en una cadena de suministro más competitiva.

La plataforma está diseñada para enfrentar tres barreras fundamentales que enfrentan las PYMES en Latinoamérica y el Caribe a la hora de realizar comercio en el exterior y son:

- Falta de acceso a clientes confiables

- Información limitada sobre la internacionalización y requisitos para ingresar a las cadenas de suministro competitivas

- Poco acceso al financiamiento
Además de esto, es importante mencionar que Connect Americas fue creada por el Banco Interamericano de Desarrollo (BID) y cuatro socios clave:

- Google

- DHL

- Visa

- Alibaba

La plataforma fue creada en marzo del 2014, y desde entonces ya ha llegado a más de 1 millón de usuarios, registrándose más de 60 mil empresas reportándose transacciones por más de 112 millones de dólares estadounidenses.

A parte de esto, la plataforma recopila MOOC gratuitos a los cuales todos los usuarios pueden registrarse, a través de la plataforma ya se han registrado más de 15 mil usuarios a estos cursos.

Para brindar el mayor apoyo posible a las PYMES que desean crecer, la plataforma permite a los empresarios y emprendedores conectarse con otros empresarios y emprendedores a través de tres herramientas:

- Conéctate

Los usuarios pueden promocionar sus empresas a comunidades y personas, además, pueden encontrar oportunidades de negocio y acudir a ferias empresariales para promover sus productos o servicios

- Aprende

Mediante esta función, los usuarios pueden obtener información acerca de casos de éxito, asesoría para empresas, guías, consejos y academia la cual contiene una amplia gama de material de estudio para fortalecer las capacidades de los empresarios de las PYMES.

\section{- Finanzas}

Usando esta función, los usuarios pueden tener acceso a las oportunidades de financiamiento ofrecidos en sus países de origen por entidades gubernamentales y no gubernamentales, que no son promovidos a través de los medios de comunicación masivos. 


\section{Conclusiones}

El reto fundamental para que las PYMES sobrevivan en el mercado es, vender más, y esperamos que usen estas herramientas para que aumenten sus ventas y puedan mantenerse en el mercado.

Todas las herramientas que hemos expuesto en este artículo ya han sido implementadas con éxito en numerosas empresas del país cuya información está registrada en las bases de datos de las entidades gubernamentales mencionadas en el artículo, y en cuanto a la red social empresarial "Connect Americas" numerosas PYMES la están usando exitosamente, consiguiendo socios en otros países y nuevos clientes para sus productos o servicios.

Ahora que hemos recopilado los principales retos para la supervivencia[14][15] de las PYMES en Colombia, solo nos queda enviarles a los empresarios de las PYMES colombianas, y de toda Latinoamérica un mensaje de ánimo y esperanza, para que construyan la base que sostendrá la economía de nuestros países y familias en el futuro[16], que a través de las herramientas que están al alcance de todos edifiquen la prosperidad y estabilidad que se merecen los empresarios y emprendedores de toda Latinoamérica.

\section{REFERENCIAS}

[1] ACOPI. Revista Mi Pyme + Productiva. Obtenido de Revista Mi Pyme + Productiva: https:// acopi.org.co/revista-mi-pyme-mas- productiva/. 2017.

[2] M. Franco Ángel \& D. Urbano, Dinamismo de las PYMES en Colombia: un estudio comparativo de empresas. Recuperado el 28 de Agosto de 2017, de Dinamismo de las PYMES en Colombia: un estudio comparativo de empresas: http://www. redalyc.org/articulo.oa?id=2903126500 9. 2014.

[3] J. F. Suárez, Los problemas que agobian a las pequeñas y medianas empresas. Recuperado el 18 de Septiembre de 2017, de Los problemas que agobian a las pequeñas y medianas empresas: http:/ / www.elcolombiano.com/negocios/empresas/c apital-y-mano-de-obra-agobian-a-pymes-paisasIJ6496684. 2017.

[4] Confecámaras. Gobierno Corporativo. Obtenido de Gobierno Corporativo: http://gc.confecamaras. co/front/. 2012.
[5] Confecámaras. ¿Qué es Gobierno Corporativo? Obtenido de ¿Qué es Gobierno Corporativo?: http:/ / www.confecamaras.org.co/gobierno- corporativo/165-que-es-gobierno-corporativo. 2016.

[6] Grupo Bancolombia. ¿Qué es el Factoring? Obtenido de ¿Qué es el Factoring?: https:/ / www.youtube. com/watch?v=5AUkOm4dO_8. 2011.

[7] N. Hill, N. Piense y hagase rico. México: Grupo Editorial Tomo S.A. 2004.

[8] AMCHAM. Cámara de comercio colombo americana. Recuperado el 18 de Septiembre de 2017, de Cámara de comercio colombo americana: http:/ / www.amchamcolombia.co/. 2017.

[9] Procolombia. Ruta exportadora. Obtenido de Ruta exportadora: http://www.procolombia.co/rutaexportadora/. 2017.

[10] Confecámaras. Beneficios del Gobierno Corporativo. Obtenido de Beneficios del Gobierno Corporativo: http:/ / www.confecamaras.org.co/cooperacion-ycompetitividad/gobierno-corporativo/167- beneficios-del-gobierno-corporativo. 2016

[11] CCCB. Cámara de comercio colombo Británica. Recuperado el 18 de Septiembre de 2017, de Cámara de comercio colombo Británica: http://www. colombobritanica.com/web/. 2017.

[12] ConfeCámaras. Determinantes de la supervivencia empresarial en Colombia. Recuperado el 18 de Septiembre de 2017, de: http://www. confeca maras.org.co/phocadownload/Cuader nos_de analisis_economico/Cuaderno_de_An \% D0\% B0lisis_Economico_N_14.pdf. 2017.

[13] A. Murga, ¿Networking? Obtenido de ¿Networking?: http:/ / negociosynetworking.net, 2015.

[14] Departamento Nacional de Planeación. CONPES 3866 POLÍTICA NACIONAL DE DESARROLLO PRODUCTIVO . Recuperado el 18 de Septiembre de 2017, de CONPES 3866 POLÍTICA NACIONAL DE DESARROLLO PRODUCTIVO: http://www. colombiacompetitiva.gov.co/prensa/inf ormes/ Conpes-3866-de-2016-Politica-desarrollo- productivo. pdf. 2016.

[15] Dinero. ¿Por qué están pesimistas las pymes en Colombia? Recuperado el 28 de Agosto de 2017, de ¿Por qué están pesimistas las pymes en Colombia?: http://www.dinero.com/edicion- impresa/ pymes/articulo/percepciones-de-los- empresarios-pyme-en-colombia/241660. 2017.

[16] Dinero. ¿Por qué las mipymes están relegadas del mercado exportador? Recuperado el 28 de Agosto de 2017, de ¿Por qué las mipymes están relegadas del mercado exportador?: http://www.dinero. com/economia/articulo/exportaci ones-de-lasmipymes-en-colombia/241893. 2017.

Rev. Ingeniería, Matemáticas y Ciencias de la Información Vol. 6 / Núm. 11 / enero - junio de 2019; pág. 113-120 